\title{
TRANSTORNO DO ESPECTRO AUTISTA: ATUALIZAÇÃO
}

Verônica Ribeiro Possamai. Transtorno do Espectro Autista: atualização. Revista Saúde Dinâmica, vol. 3, núm. 2, 2021. Faculdade Dinâmica do Vale do Piranga. 


\title{
Transtorno do Espectro Autista: atualização
}

\author{
Autistic Spectrum Disorder: update \\ Verônica Ribeiro Possamai \\ Fonoaudióloga. Mestre em Bioética, Ética Aplicada e Saúde Coletiva pela Universidade Federal do Rio de Janeiro. \\ Doutoranda no Programa de Pós-graduação em Bioética, Ética Aplicada e Saúde Coletiva (PPGBIOS) pela UFRJ. \\ Fonoaudióloga do Centro de Atenção Psicossocial Infantil Pequeno Hans (CAPSi). \\ Autor correspondente: vrpossamai.fono@gmail.com
}

\section{Resumo}

O Transtorno do Espectro Autista é caracterizado por distúrbios na linguagem e na interação social, além de interesses e comportamentos restritos e repetitivos. É possível observar o número crescente de diagnósticos que, apesar de diversos motivos controversos, tornam o autismo uma questão de saúde pública. Este estudo tem como objetivo analisar os maiores desafios enfrentados pelos autistas e seus familiares na Atenção Básica de Saúde. Segundo o levantamento bibliográfico, os maiores desafios encontrados estão baseados na falta de conhecimento dos profissionais de saúde sobre o transtorno, acarretando na identificação e diagnóstico tardio e, consequentemente uma demora na intervenção terapêutica. Na maioria dos casos, os profissionais da Atenção Primária fazem parte do primeiro contato do paciente no Sistema Único de Saúde e a falta de conhecimento dos mesmos acerca do autismo acarreta em dificuldades na orientação das famílias, equipes que o acompanham e nas intervenções necessárias. A identificação precoce permite uma intervenção adequada nos primeiros anos de vida, nos quais ocorrem melhor aproveitamento e desenvolvimento de atividades funcionais devido a neuroplasticidade. Quando isso não ocorre, anos de intervenções terapêuticas são perdidos, dificultando o desenvolvimento da criança. Diante do estudo realizado, observamos a necessidade de ampliar a qualificação e conhecimento dos profissionais de saúde da Atenção Primária.

Palavras-chave: Atenção básica; Transtorno do Espectro Autista; Atenção Primária; Sistema Único de Saúde.

\begin{abstract}
Autistic Spectrum Disorder is characterized by disorders in language and social interaction, in addition to restricted and repetitive interests and behaviors. It is possible to observe the growing number of diagnoses that, despite several controversial reasons, make autism a public health issue. This study aims to analyze the biggest challenges faced by autists and their families in Primary Health Care. According to the bibliographic survey, the greatest challenges encountered are based on the lack of knowledge of health professionals about the disorder, resulting in late identification and diagnosis and, consequently, a delay in therapeutic intervention. In most cases, Primary Care professionals are part of the patient's first contact with the Unified Health System and their lack of knowledge about autism causes difficulties in guiding families, teams that accompany them and in the necessary interventions. Early identification allows an adequate intervention in the first years of life, in which better use and development of functional activities occur due to neuroplasticity. When this does not happen, years of therapeutic interventions are lost, hindering the child's development. In view of the study carried out, we observed the need to expand the qualification and knowledge of primary care health professionals.

Key words: Basic Care; Autism; Autistic Spectrum Disorder; Primary Care; Unified Health System.
\end{abstract}




\section{INTRODUÇÃO}

O Transtorno do Espectro Autista (TEA) é classificado como uma doença mental, caracterizada por déficits na linguagem e interação social, bem como interesses e comportamentos restritos e repetitivos. Geralmente, os primeiros sinais podem aparecer no primeiro ano de vida, em casos mais graves (APA, 2014). Este transtorno traz com ele muitas dificuldades e exige acompanhamento profissional para os pacientes e suas famílias. O número de diagnósticos vem crescendo a cada ano, preocupando os profissionais. Atualmente, nos EUA, os estudos indicam que 1\% das crianças são autistas (APA, 2014; KIM et al., 2011; RICE et al., 2012). Este aumento é controverso, já que alguns autores (APA, 2014; GRAF, 2017, KIM et al., 2011, RICE, et al., 2012) atribuem a alguns fatores: mudança dos critérios diagnósticos, que, atualmente, amplia o diagnóstico para outras síndromes que antes não faziam parte do espectro; maior número de crianças diagnosticadas; maior presença dos autistas no meio social; e o aumento real dos casos (GRAF, 2017). Devemos olhar o TEA como um problema de saúde pública, no qual observamos alguns problemas, principalmente na Atenção Primária à Saúde (APS), onde é realizado o primeiro contato desses pacientes para atendimento no Sistema Único de Saúde (MENDES, 2011).

A falta de conhecimento dos profissionais de saúde sobre o autismo foi um dos problemas identificados no presente estudo, causando informações equivocadas e dificuldade de adesão ao tratamento por falta de orientações. Algo importante que é desencadeado pela falta de conhecimento é a identificação tardia dos sinais de risco para o autismo, e, por consequência, ocasionando o mesmo com o diagnóstico (SURMEN et al., 2015; TIMLIN et al., 2015; VASCONCELLOS; CASTIEL, 2020). Quando isso ocorre, a criança perde anos de possíveis intervenções, que poderiam diminuir as perdas funcionais ocasionadas pelo transtorno (BRASIL, 2014; WU, 2020). Após a identificação dos sinais e o diagnóstico, a mesma deve ser encaminhada para intervenção terapêutica. Esta pode ser realizada a partir de dois campos: reabilitação e saúde mental. Ambas realizam atendimento multiprofissional às crianças com autismo, mas acabam por entrar em conflito em relação ao conhecimento do transtorno, dificultando o acesso ao tratamento (OLIVEIRA et al., 2017). Logo, foi possível identificar algumas dificuldades enfrentadas pelos autistas e suas famílias que vão além do diagnóstico em 
si, como o acesso à saúde e ao tratamento, bem como o desconhecimento dos profissionais e da sociedade acerca do transtorno.

Diante dos problemas expostos, compreende-se o desafio que as famílias precisam enfrentar, pois além de lidar com o diagnóstico e todas as dificuldades que ele acarreta, são pessoas em situação de vulnerabilidade, e ao buscar acesso aos seus direitos básicos, como saúde e educação, encontram problemas que dificultam ainda mais sua dignidade. Desta forma, existe a necessidade de um olhar diferenciado para essa população, a partir de uma abordagem bioética, para valorizar sua vulnerabilidade e tentar propor um cuidado adequado.

Foi realizado um levantamento bibliográfico nas bases de dados Biblioteca Virtual da Saúde (BVS), National Center for Biotechnology Information (PUBMED) e Scientific Electronic Library Online (SCIELO) com os descritores "Atenção básica”, "Transtorno do Espectro Autista", “Atenção Primária” e "Sistema Único de Saúde”. Após a busca, foram inclusos artigos por relevância do título e abstract. Também foram incluídos documentos oficiais e leis importantes para o tema. O objetivo do presente artigo é apresentar o Transtorno do Espectro Autista e suas características principais, bem como as configurações do Sistema Único de Saúde, principalmente o campo da Atenção Primária à Saúde, analisando os maiores desafios enfrentados por estes indivíduos.

\section{CARACTERÍSTICAS GERAIS DO TRANSTORNO DO ESPECTRO AUTISTA}

O TEA é um transtorno mental caracterizado por alterações comportamentais e é classificado por grau de severidade, baseando-se nos seguintes critérios: prejuízos na comunicação social, e padrões restritos e repetitivos de comportamento (APA, 2014).

O primeiro estudo acerca do TEA foi realizado em 1943, no Hospital John Hopkins, pelo médico Leo Kanner. Ele avaliou 11 crianças que apresentavam, principalmente, problemas para se relacionar desde o nascimento, além de outros comprometimentos, como: maneirismos motores, resistência a mudanças, e alterações linguísticas. Kanner definiu como "distúrbios autísticos de contato afetivo", além de criar o termo "solidão autística" para representar o isolamento social extremo (KLIN, 2006; ZAMPIROLI; SOUZA, 2012). 
Atualmente são utilizados dois grandes sistemas de critérios preliminares de diagnósticos, são eles: Manual diagnóstico e estatístico de transtornos mentais (DSM-5), da Associação Americana de Psiquiatria (APA), de 2013; e a Classificação Internacional de Doenças (CID-10), da Organização Mundial da Saúde (OMS) (FREITAS, 2019).

No CID-10, o autismo infantil é um dos transtornos classificados como transtornos globais do desenvolvimento (TGD). Os TGD incluem o autismo infantil; autismo atípico; síndrome de Rett; outro transtorno desintegrativo da infância; transtorno com hipercinesia associada a retardo mental e movimentos estereotipados; síndrome de Asperger; outros transtornos globais do desenvolvimento; e transtornos globais não especificados do desenvolvimento. Em junho de 2018, a OMS lançou o CID-11. Nesta edição o autismo é classificado através da associação com deficiência intelectual e comprometimento da linguagem funcional. Desta forma a classificação fica mais coerente com o DSM-5, incluindo os TGD (inclusive síndromes de Asperger e Rett) dentro da categoria de TEA (APA, 2014; OMS, 2003, 2018).

O Manual de Diagnósticos e Estatísticas de Desordens Mentais ou Diagnostic and Statistical Manual of Mental Disorders (DSM) que foi desenvolvido pela APA, define e classifica os transtornos mentais e foram lançadas cinco edições. O autismo foi citado pela primeira vez pela APA no DSM III e atualmente está definido como Transtorno do Espectro Autista no DSM-5 (RAPIN; TUCHMAN, 2009). Neste manual o nível de gravidade é classificado de acordo com o nível de apoio necessitado, gerando 3 níveis: o nível 1 que corresponde ao autista que necessita de apoio, o nível 2 que corresponde ao autista que necessita de apoio substancial, e o nível 3 que corresponde ao autista que necessita de apoio muito substancial (APA, 2014).

Os sintomas do TEA causam prejuízos no ambiente social, ocupacional, e em outras áreas, podendo ocorrer também, deficiência intelectual associada. Os sinais começam a ser percebidos a partir de um ano de idade. Porém, se os comprometimentos forem muito graves, podem ser vistos no primeiro ano de vida. Se os sintomas forem mais sutis, podem ser percebidos após dois anos. Os principais sinais são os atrasos no desenvolvimento e as perdas sociais e linguísticas. Em alguns casos, conhecidos como autismo regressivo, podem haver perdas de habilidades, como por exemplo a fala e a interação, ou seja, após a criança adquirir 
esta capacidade, ela perde gradualmente. Como essa regressão é algo raro em outras síndromes, torna-se um grande sinal para o diagnóstico de autismo (APA, 2014).

A etiologia do TEA ainda é desconhecida, porém estudos indicam possibilidades ambientais (idade parental avançada, baixo peso ao nascer, exposição ao ácido valpróico) ou genética/fisiológica (hereditariedade, mutação genética). A proporção do diagnóstico do autismo é quatro vezes maior no sexo masculino que no feminino. Porém, em meninas existe maior propensão a ter deficiência intelectual como comorbidade (APA, 2014).

As intervenções terapêuticas devem ser baseadas em teorias sólidas, metodologias rigorosas e com eficácia comprovada cientificamente, com objetivo de minimizar os déficits (comunicação social e comportamentos restritos e repetitivos), proporcionar maior independência funcional através da facilitação da aprendizagem e habilidades adaptativas e diminuir, eliminar ou evitar problemas comportamentais que dificultem suas habilidades funcionais (HYMAN et al., 2020).

Os indivíduos com TEA necessitam de tratamento por um grande tempo de suas vidas. São necessárias intervenções médicas, comportamentais e de desenvolvimento, bem como suporte de assistência social. A criança deve ser atendida por uma equipe multidisciplinar nos âmbitos da saúde médica a mental, terapêutica, educacional e de prestação de serviços sociais e comunitários. É essencial que a equipe de profissionais permaneça alinhada com a família para continuidade e eficácia do tratamento. O plano de tratamento deve ser individualizado para que respeite a singularidade de cada indivíduo.

Cabe ressaltar que existe um movimento chamado "neurodiversidade", que é composto, principalmente, por autistas de alto funcionamento, ou síndrome de Asperger. As pessoas que integram este movimento, afirmam que o autismo é uma característica humana, não necessitando de tratamento. Ao contrário dos familiares de autistas de "baixo funcionamento", que entendem o transtorno com uma doença e compreendem que os mesmos não têm condições de se autodefender. Os integrantes do movimento negam a concepção que o autismo é uma doença, por isso também negam sua cura, se distanciando do modelo biomédico e se aproximando no modelo social da deficiência (SADZINSKI JR et al., 2020). Este paradigma favorece um olhar que ultrapassa o que foi marcado pela ótica clínica e médica (VIANA; MANRIQUE, 2020). 


\section{O TEA COMO UM PROBLEMA DE SAÚDE PÚBLICA}

Algo muito discutido acerca do TEA é o visível aumento dos números de casos diagnosticados. Diversas hipóteses são levantadas como aumento da identificação dos casos, mais divulgação do transtorno, inclusão dos autistas na sociedade, inclusão de algumas síndromes no espectro, e o aumento de fato do número de pessoas com o transtorno (GRAF, 2017).

O autismo era considerado um transtorno raro, com cerca de 1 caso em 2000. A partir da década de 90, os sistemas de saúde do EUA começaram a se preocupar com o aumento dos diagnósticos. Diversos estudos, utilizando a Classificação Internacional de Doenças (CID-10) e o Manual Estatístico de Doenças Mentais (DSM-5), identificaram um aumento dos números de casos para seis ou sete em 1000 (0,6 ou 0,7\%) (RICE et al., 2012).

Com o número crescente de diagnósticos, se torna necessário disponibilizar meios para que esta população tenha qualidade de vida. Os autistas estão ocupando mais espaços sociais e necessitam ter os mesmos direitos básicos do restante da população, como saúde e educação de qualidade (POSSAMAI, 2016). Na infância, eles podem apresentar dificuldade de socialização e aprendizagem, criando dificuldades no contexto escolar. Na vida adulta, pode haver dificuldade de manter a autonomia, principalmente em casos mais graves. Existem estudos que apresentam os prejuízos nas condições psicológicas dos indivíduos autistas diante das dificuldades enfrentadas devido ao transtorno, inclusive citando muitos casos de ansiedade e depressão, e até tentativa de suicídio (CASSIDY, 2018).

\section{(DES)CONHECIMENTO SOBRE O AUTISMO}

Apesar de o TEA ser um transtorno que começou a ser estudado há poucas décadas, existem muitos estudos sendo desenvolvidos para se obter mais conhecimento e, consequentemente, promover melhores atendimentos para esses indivíduos. É necessário que os profissionais que os atendem estejam qualificados para que seja criado um projeto terapêutico adequado, bem como sejam realizadas as orientações adequadas às famílias. 
Com o crescimento da prevalência do transtorno, surgiram também informações equivocadas que prejudicam a saúde da população. Na década de 50 e 60, existiu a teoria da "mãe geladeira", de John Watson, que afirmava que os pais não eram suficientemente responsivos com seus filhos (KLIN, 2006). O diagnóstico de autismo mexe com a estrutura familiar, a culpabilização dos pais piora extremamente a forma de lidar com o transtorno.

Outro fato que se tornou um grande problema de saúde pública desencadeado pela falta de conhecimento sobre o TEA é o movimento antivacina. Um estudioso chamado Andrew Wakefield publicou um artigo, em 1998, com o título de "MMR vaccination and autism" na revista "The Lancet”, no qual associava a vacina tríplice com o autismo. Após investigação, foi identificado conflito de interesse na pesquisa, associado ao lançamento de uma nova vacina para o sarampo. O General Medical Council - GMC cassou o registro profissional do pesquisador e a revista retratou o artigo. Apesar de ter sido retratado, o pesquisador ter sofrido as consequências, e terem sido realizadas diversos incentivos à vacinação, uma parte da população foi influenciada pela suspeita e optou por não vacinar, aumentando o número dos casos sarampo (VASCONCELLOS; CASTIEL, 2020).

Os fatos anteriormente citados demonstram a importância de os profissionais possuírem conhecimento sobre o TEA, para que sejam promovidas orientações e intervenções eficazes para os familiares e pacientes, além de combater as informações incorretas que somam problemas para a saúde da população.

Surmen et al. realizaram um estudo, em 2015, no qual entrevistou 160 adultos no Centro de Saúde da Família em Istambul com objetivo de mensurar o nível de conhecimento sobre o autismo e suas atitudes diante desses indivíduos. Utilizou-se, como ferramenta, um questionário baseado nas características descritas no DSM-5. Apenas 38,8\% dos entrevistados tinham ouvido falar a palavra "autismo". Neste estudo também foram questionadas as reações dos participantes diante do convívio com pessoas autistas. A maior parte das respostas indicam receptividade e ausência de discriminação no ambiente escolar e de trabalho, porém afirmam que sentem pena e tentam ajuda-los em tarefas do cotidiano. Os autores concluem que, apesar da maioria das respostas obtidas sobre o convívio ser positivas, menos da metade dos entrevistados tinham ouvido a palavra "autismo". Por isso se faz necessário que seja intensificada a educação em saúde para realizar conscientização sobre o transtorno. 
Timlin et al. (2015) pesquisaram sobre a adesão de adolescentes autistas ao tratamento através de uma revisão sistemática de literatura. Um dos fatores que interferem na adesão dos mesmos é a compreensão da importância da continuidade do tratamento, seja ele medicamentoso e/ou terapêutico. Para isso é necessário que o adolescente seja bem orientado por um profissional qualificado sobre o transtorno.

Diante do exposto, observamos a necessidade de ampliar o conhecimento sobre o transtorno na sociedade e, principalmente, entre os profissionais da saúde e educação que trabalham com estes indivíduos. Desta forma, será possível evitar a divulgação de informações incorretas, além de possibilitar a identificação precoce dos sinais indicadores do TEA, permitindo a intervenção precoce.

\section{TRIAGEM E DIAGNÓSTICO PRECOCE}

Diversos estudos afirmam que o transtorno pode ser identificado a partir dos 16 meses de idade, porém a idade média de diagnóstico, nos EUA, é aos 4 ou 5 anos, podendo ser mais tardia em populações de baixa renda e moradoras de zona rural (GUTHRIE, 2019 et al., SCHRADER et al., 2020). A “American Academy of Pediatrics" (AAP) recomenda que a triagem seja realizada entre os 18 e 24 meses para ser dado início à intervenção (DOWNS et al., 2019; SCHRADER, 2020; WU, 2020; MARLAND et al., 2019).

É de extrema importância que a identificação dos sinais de TEA seja feita o mais precocemente possível, pois, dessa forma, podem ser realizadas as intervenções necessárias em uma fase sensível e privilegiada do desenvolvimento devido à neuroplasticidade presente nos primeiros anos de vida e com objetivo de proporcionar experiências essenciais de vida de um bebê. Entretanto, deve haver cuidado com o diagnóstico precipitado que pode influenciar no desenvolvimento da criança, além de haver a possibilidade de o diagnóstico de TEA ofuscar a real dificuldade (BRASIL, 2014; WU, 2020).

Existem diversos instrumentos de triagem e escalas padronizadas para que os primeiros sinais sejam identificados e assim mantenham um acompanhamento terapêutico e avaliativo. Os instrumentos indicados pela cartilha de Diretrizes de Atenção à Reabilitação da Pessoa com Transtornos do Espectro do Autismo (TEA) são o "Indicadores Clínicos de Risco para o Desenvolvimento Infantil" (IRDI) para uso na Atenção Básica e o Modified Checklist for 
Autism in Toddlers (M-CHAT) que é de uso livre por todo o Brasil (BRASIL, 2014). Schrader et al. (2020) salientam a importância do médico da atenção básica na triagem, diagnóstico e intervenção dos pacientes autistas.

Marland et al. (2019) defendem a utilização de testes rápidos para que sejam mais aderidos às consultas primárias e assim seja feita uma identificação rápida dos sinais de autismo, mesmo que não sejam utilizados como testes de diagnóstico. Segundo os autores, esse tipo de avaliação se torna acessível para ser utilizada em regiões de baixa renda. Existem alguns estudos que afirmam a ineficácia do M-CHAT como teste de triagem devido sua baixa sensibilidade e defendem a necessidade de desenvolver novos testes para melhor identificação (GUTHRIE et al., 2019; SCHRADER et al., 2020).

É importante ressaltar que os testes de triagem não têm função diagnóstica. Eles identificam crianças que apresentam sinais indicativos de autismo e o profissional e/ou equipe que o acompanha realiza o encaminhamento para testes com objetivo diagnóstico. Alguns autores afirmam que devem ser feitos testes de triagem regularmente em toda criança atendida na atenção primária (GUTHRIE et al., 2019; HYMAN et al., 2020), bem como realizar um registro eletrônico para maior eficácia do diagnóstico. (HYMAN et al., 2020) Essas crianças, frequentemente, são identificadas pela primeira vez por um pediatra, médico da família, pais ou cuidador (BRYAN; ZWAIGENBAUM; IP, 2019).

Hyman cita algumas dificuldades na triagem de crianças com autismo na atenção básica. Ela relata sobre o fato de crianças com autismo de grau leve ser diagnosticados apenas em idade escolar, quando os sintomas linguísticos e de interação começam a prejudicar sua vida diária. Outro desafio citado pela autora é a dificuldade de compreensão e confiança dos pais de diferentes culturas e línguas, por isso os profissionais devem ser bem treinados para atender a toda a população (HYMAN, 2020).

Em um estudo de Ebert, Lorenzini e Silva (2015), dez mães foram entrevistadas com objetivo de saber sobre suas percepções quanto a alterações no comportamento e/ou desenvolvimento de seus filhos e suas trajetórias na busca pelo diagnóstico. Porém, afirmaram que os profissionais não percebiam alterações no comportamento das crianças, dificultando o diagnóstico. Algumas participantes da pesquisa afirmaram que as primeiras observações foram feitas pelos pais da criança e por profissionais da área de educação (professores, coordenadores, psicopedagogos). Desta forma, as mães passam por uma peregrinação até a identificação dos 
sinais do transtorno e, consequentemente, o diagnóstico e a intervenção terapêutica, que acaba por acontecer tardiamente, perdendo grande potencial de evolução.

Após a triagem, se identificados os sinais indicativos de autismo, a criança deve ser encaminhada para avaliação diagnóstica. Devem ser analisadas as características de linguagem, cognição, habilidades sensoriais e adaptativas. Esta avaliação deve ser detalhada, a partir de vários encontros e conversa com os pais, para que não haja falsos positivos ou negativos (HYMAN, 2020).

\section{INTERVENÇÃO TERAPÊUTICA}

Apesar de haver poucos estudos abordando esse tema, é importante falar sobre o acesso dos pacientes às abordagens terapêuticas, principalmente no Sistema Único de Saúde.

\footnotetext{
"A articulação com outros pontos de atenção deve ser feita institucionalmente, de maneira a evitar que as famílias se desloquem desnecessariamente e tentem individualmente seu acesso a outros pontos de atenção que se façam necessários" (BRASIL, 2015, p. 95).
}

Existem dois documentos oficiais para orientação do cuidado às pessoas autistas, são eles: 'Diretrizes de Atenção à Reabilitação da Pessoa com Transtorno do Espectro do Autismo (TEA)' e Linha de Cuidado para a Atenção às pessoas com transtornos do espectro do autismo e suas famílias na rede de atenção psicossocial do Sistema Único de Saúde’, representando dois campos de atuação referentes à abordagem diagnóstica e terapêutica dos indivíduos com autismo. O primeiro documento é utilizado pelo campo de atuação conhecido como "reabilitação", inclui o TEA no grupo de deficiências, possui uma abordagem mais objetiva e "nasceu" das associações de pais de crianças com o transtorno, enquanto o segundo documento é utilizado pela atenção psicossocial, aborda como um transtorno mental, possui uma abordagem mais subjetiva, e teve origem nos movimentos da luta antimanicomial (OLIVEIRA, et al., 2017; SILVA; FURTADO, 2019; POSSAMAI, 2021). Estes dois campos entram em discordâncias que acabam gerando conflitos teóricos concentrados em torno de uma reivindicação sobre qual dos dois grupos possuiria mais conhecimento e legitimação acerca do 
autismo, e assim, consequentemente, os principais autores das políticas públicas (OLIVEIRA et al., 2017).

Estas duas esferas de atuação com o usuário autista do SUS possuem convergências e divergências. O PTS (Planejamento Terapêutico Singular) é uma convergência entre elas, pois ambas afirmam a importância de um documento criado com a família, o usuário e os profissionais que os atendem, com o objetivo de planejar seu cuidado a partir da identificação de suas necessidades e singularidades (VALERIO et al., 2020). Oliveira et al. (2017) exemplificou algumas divergências que são relacionadas, principalmente, às diretrizes diagnósticas e as diretrizes para o cuidado. As diretrizes diagnósticas do campo da reabilitação são de caráter técnico e objetivo, enquanto do campo da atenção psicossocial são mais subjetivas, apresentando possíveis vetores culturais, éticos e políticos. As diretrizes para o cuidado no campo da reabilitação visam o desenvolvimento das habilidades funcionais, enquanto no campo da atenção psicossocial visam a ampliação dos laços sociais de cada usuário.

Segundo as Diretrizes de Atenção à Reabilitação da Pessoa com Transtornos do Espectro do Autismo (TEA), os locais que oferecem atenção especializada à saúde são: os Centro Especializado em Reabilitação (CER), Serviços de Reabilitação Intelectual e Autismo, Centro de Atenção Psicossocial (CAPS), outros institutos, ambulatórios e especialidades (BRASIL, 2014).

\section{CONSIDERAÇÕES FINAIS}

Através deste estudo, foi possível observar que os maiores desafios enfrentados pelos indivíduos autistas e suas famílias na APS são acarretados, principalmente, pela falta de conhecimento acerca do transtorno. Isso interfere no percurso no Sistema Único de Saúde, já que o atendimento na APS, geralmente, consiste no primeiro contato dos pacientes na saúde pública. Desta forma, o transtorno é identificado tardiamente e, consequentemente, há um diagnóstico e intervenção tardia. A identificação precoce é essencial para que haja intervenção nos primeiros anos de vida, nos quais há um melhor aproveitamento neuronal dos estímulos devido à neuroplasticidade. Apesar de haver uma ferramenta de identificação, ainda é insuficiente, já que muitas crianças são encaminhadas para o serviço de saúde somente em idade 
escolar, quando são observados os sinais indicativos do autismo pelos profissionais da educação. É necessária uma observação criteriosa e um maior conhecimento sobre o TEA, para que os sinais iniciais sejam identificados, sendo possível encaminhar para uma avaliação diagnóstica e para a intervenção terapêutica adequada nos anos iniciais de vida. Como exposto anteriormente, o conhecimento acerca do autismo é extremamente necessário, desta forma, este estudo contribuiu para apresentar questões bioéticas envolvidas no percurso do usuário autista no Sistema Único de Saúde e assim se tornar mais um instrumento de divulgação acerca do transtorno. Quanto as limitações, se faz necessária uma pesquisa empírica com os profissionais da saúde para identificar possíveis dificuldades na identificação do autismo, o que não foi possível realizar neste estudo. Por isso, é notória a necessidade de mais estudos com este tema, abordando outras questões referentes às dificuldades da identificação precoce.

\section{REFERÊNCIAS}

APA Manual diagnóstico e estatístico de transtornos mentais: DSM-5 [American Psychiatric Association; tradução: Maria Inês Corrêa Nascimento... et al.]; 5. ed. Porto Alegre: Artmed, 2014.

BRASIL. Ministério da Saúde. Secretaria de Atenção à Saúde. Departamento de Ações Programáticas Estratégicas. Diretrizes de Atenção à Reabilitação da Pessoa com Transtornos do Espectro do Autismo (TEA). Ministério da Saúde, Secretaria de Atenção à Saúde, Departamento de Ações Programáticas Estratégicas. - Brasília: Ministério da Saúde, 2014. 86p.

. Ministério da Saúde. Secretaria de Atenção à Saúde. Departamento de Atenção Especializada e Temática. Linha de cuidado para a atenção às pessoas com transtornos do espectro do autismo e suas famílias na Rede de Atenção Psicossocial do Sistema Único de Saúde / Ministério da Saúde, Secretaria de Atenção à Saúde, Departamento de Atenção Especializada e Temática. - Brasília: Ministério da Saúde, 2015. 156 p.

BRYAN, J.A. ZWAIGENBAUM, L. IP, A. Standart of diagnostic assessment for autism spectrum disorder. Paediatrics \& Child Health, v. 24, n. 7, p. 444-451, 2019. DOI: https://doi.org/10.1093/pch/pxz117.. Disponível em: https://academic.oup.com/pch/article/24/7/444/5603338. Acesso em: 12 de novembro de 2020.

CASSIDY, S. et al., Risk markers for suicidality in autistic adults. Molecular Autism. v. 9, n. 42, p.1-14, 2018. DOI: 10.1186/s13229-018-0226-4.. Disponível em:

https://www.ncbi.nlm.nih.gov/pmc/articles/PMC6069847/. Acesso em: 13 de novembro de 2020. 
DOWNS, S. M. et al., Effect of a computer- based decision support interventionon autism spectrum disorder screening in pediatric primary care clinics: a cluster randomized clinical trial. JAMA Network Open. v. 2, n. 12, p. 1-12, 2019. DOI:

10.1001/jamanetworkopen.2019.17676.. Disponível em:

https://www.ncbi.nlm.nih.gov/pmc/articles/PMC6991212/. Acesso em: 12 de novembro de 2020.

EBERT, M; LORENZINI, E; SILVA, E. F. Mães de crianças com transtorno autístico: percepções e trajetórias. Revista Gaúcha de Enfermagem, v. 36, n. 1, p. 49-55, 2015. DOI: https://doi.org/10.1590/1983-1447.2015.01.43623.. Disponível em:

http://www.scielo.br/scielo.php?script=sci_arttext\&pid=S198314472015000100049\&lng=en\&nrm=iso. Acesso em: 11 de novembro de 2020.

FREITAS, E. Transtornos do neurodesenvolvimento: conhecimento, planejamento e inclusão real. Rio de Janeiro: Wak Editora, 2019. 184p.

GRAF, W. et al., The autism "epidemic": ethical, legal and social issuesin a developmental spectrum disorder. American Academy of Neurology. v. 4, n. 88, p.1-10, 2017. DOI:

10.1212/WNL.0000000000003791. Disponível em:

https://pubmed.ncbi.nlm.nih.gov/28275086/. Acesso em: 10 de novembro de 2020.

GUTHRIE W et al., Accuracy of Autism Screening in a Large Pediatric Network. Pediatrics, v. 144, n. 4, p. 1-12 .2019. DOI: https://doi.org/10.1542/peds.2018-3963. Disponível em: https://pediatrics.aappublications.org/content/144/4/e20183963\#: :text=RESULTS\%3A\%20 Nearly\%20universal\%20screening\%20was,PPV\%20was\%20lower\%20in\%20girls. Acesso em: 13 de novembro de 2020.

HYMAN, S.L. et al., Identification, evaluation, and management of children with autism spectrum disorder. Pediatrics. v. 145, n. 1, p. 1-64, 2020. DOI:

https://doi.org/10.1542/peds.2019-3447. Disponível em:

https://pediatrics.aappublications.org/content/145/1/e20193447. Acesso em: 15 de novembro de 2020.

KIM, Y.S et al., Prevalence of Autism Spectrum Disorders in a Total Population Sample.

American Journal of Psychiatry. v. 168, n. 9, p. 904-12, 2011. DOI:

10.1176/appi.ajp.2011.10101532. Disponível em:

https://pubmed.ncbi.nlm.nih.gov/21558103/. Acesso em: 10 de novembro de 2020.

KLIN, A. Autismo e síndrome de Asperger: uma visão geral. Revista Brasileira de

Psiquiatria, v. 28, p. S3-11, 2006. DOI: http://dx.doi.org/10.1590/S1516-

44462006000500002. Disponível em:

https://www.scielo.br/scielo.php?script=sci_arttext\&pid=S1516-44462006000500002. Acesso em: 10 de novembro de 2020.

MARLAND, C et al., The development of a brief screener for autism using item response theory. BMC Psychiatry, v. 19, n. 337, p. 1-8, 2019 DOI: 10.1186/s12888-019-2333-y.

Disponível em: https://www.ncbi.nlm.nih.gov/pmc/articles/PMC6829932/. Acesso em: 10 de novembro de 2020. 
MENDES, E.V. As redes de atenção à saúde. Brasília: Organização Pan-Americana da Saúde, 2011. 549p.

OLIVEIRA, B.D.C et al., Políticas para o autismo no Brasil: entre a atenção psicossocial e a reabilitação. Physis: Revista de Saúde Coletiva, Rio de Janeiro, v.27, n. 3, p. 707-726, 2017. Disponível em: https://www.redalyc.org/articulo.oa?id=400853029017. Acesso em: 14 de novembro de 2020.

OMS. ICD-11 for Mortality and Morbidity Statistics. 11 ed. 2018. Disponível em: https://icd.who.int/browse11/l-m/en\#/http://id.who.int/icd/entity/437815624.

. Classificação estatística internacional de doenças e problemas relacionados à saúde (CID-10). São Paulo: Edusp, 2003.

POSSAMAI, V.R. Autismo à luz da perspectiva dos funcionamentos. 83p. Dissertação de Mestrado. PPGBIOS, UFRJ, 2016.

. Care for autistic people in Primary Health Care: systematic review. Brazilian Journal of Development. Curitiba, v. 7, n. 1, p.11391-11410, 2021. DOI: 10.34117/bjdv7n1-777 Disponível: https://www.brazilianjournals.com/index.php/BRJD/article/view/23989/19686. Acesso em: 06 de março de 2021.

RAPIN, I; TUCHMAN, R. F. Autismo: abordagem neurobiológica. Rio de Janeiro: Artmed. 2009.

RICE, C. et al., Evaluating chances in the prevalence os the autism spectrum disorder (ASDs). Public Health Rev. v. 34, n. 2, p. 1-22, 2012. DOI: https://doi.org/10.1007/BF03391685. Disponível em: https://publichealthreviews.biomedcentral.com/articles/10.1007/BF03391685. Acesso em: 10 de novembro de 2020.

SADZINSKI JR, A et al., Neurodiversidade: levantamento das produções nacionais. Revista Eletrônica Humanitaris. v. 2, n. 2, p. 1-11, 2020. Disponível em: http://www.icepsc.com.br/ojs/index.php/revistahumanitaris/article/view/429. Acesso em: 07 de março de 2021.

SCHRADER, E et al., Integrating a New Online Autism Screening Tool in Primary Care to Lower the Age of Referral. Clinical Pediatrics. v. 59, n. 3, p. 305-309, 2020. DOI: 10.1177/0009922819900947. Disponível em: https://pubmed.ncbi.nlm.nih.gov/31976757/ Acesso em 08 de novembro de 2020.

SILVA, L.S. FURTADO, L.A.R. O sujeito autista na Rede SUS: (im)possibilidade de cuidado. Fractal Revista de Psicologia, v. 31, n. 2, p. 119-129, 2019. DOI:

https://doi.org/10.22409/1984-0292/v31i2/5635. Disponível em: https://www.scielo.br/scielo.php?script=sci_arttext\&pid=S1984-02922019000200119. Acesso em: 02 de novembro de 2020.

SURMEN, A. et al., A study exploring knowledge, attitudes and behaviours towards autism among adults applying to a Family Health Center in Istanbul. North Clinical Istanbul. v. 2, n. 1, p. 13-18, 2015 DOI: 10.14744/nci.2015.83723. Disponível em: https://pubmed.ncbi.nlm.nih.gov/28058334/. Acesso em: 05 de novembro de 2020. 
TIMLIN, U et al., Factors that Affect Adolescent Adherence to Mental Health and Psychiatric Treatment: a Systematic Integrative Review of the Literature. Scandinavian Journal of

Child and Adolescent Psychiatry and Psychology. v. 3, n. 2, p. 99-107, 2015. DOI:

10.21307/sjcapp-2015-010. Disponível em:

https://www.researchgate.net/publication/305856169_Factors that_Affect_Adolescent_Adher ence_to_Mental_Health_and_Psychiatric_Treatment_a_Systematic_Integrative_Review_of_t he Literature. Acesso em: 05 de novembro de 2020.

VALÉRIO, M. C. J et al., Educação interprofissional através do projeto terapêutico singular no PET-Saúde/ interprofissionalidade. Saúde E Meio ambiente Revista Interdisciplinar, v. 9, supl. 1, p. 55-56, 2020. DOI: 10.24302/sma.v9iSupl.1.3425. Disponível em:

https://www.researchgate.net/publication/347304129_Educacao_interprofissional_atraves_do _projeto_terapeutico_singular_no_PET-Saude interprofissionalidade. Acesso em: 05 de março de 2021.

VASCONCELOS-SILVA, P.R. CASTIEL, L.D. A internet na história dos movimentos antivacinação. ComCiência, n. 121, p. 1-4, 2010. Disponível em:

http://comciencia.scielo.br/scielo.php?script=sci_arttext\&pid=S1519-

76542010000700011\&lng=es\&nrm=iso. Acesso em: 10 de novembro de 2020.

VIANA, E.A. MANRIQUE, A. L. A neurodiversidade na formação de professores: reflexões a partir do cenário de propostas curriculares em construção no Brasil. Boletim GEPEM, n. 76, p. 91-106, 2020.. Disponível em:

http://costalima.ufrrj.br/index.php/gepem/article/view/512. Acesso em: 07 de março de 2021.

WU, C et al., The utility of the screening tool for autism in 2-year-olds in detecting autism in taiwanese toddlers who are less than 24 months of age: a longitudinal study. Journal of autism and developmental disorders. v. 50, n. 4, p. 1172-1181, 2020. DOI:

10.1007/s10803-019-04350-0. Disponível em: https://pubmed.ncbi.nlm.nih.gov/31970598/.. Acesso em: 08 de novembro de 2020.

ZAMPIROLI, W. C.; SOUZA, V. M. P. Autismo infantil: uma breve discussão sobre a clínica e o tratamento. Pediatria Moderna, v. 48, n. 4, p. 126-130, 2012. Acesso em: 10 de junho de 2020. 


\section{Declaração de Interesse}

A autora declara não haver nenhum conflito de interesse.

\section{Financiamento}

Financiamento próprio.

\section{Agradecimentos}

Agradeço à contribuição do Prof. Dr. Rodrigo Siqueira-Batista (FADIP, UFRJ e UFV), por orientar a pesquisa de doutorado à qual está vinculado o presente trabalho.

\section{Colaboração entre autores}

$O$ presente artigo foi escrito pela Verônica Ribeiro Possamai, autora que é responsável por todas as etapas de elaboração do texto 